\title{
MORTALIDAD PERINATAL EN EL HOSPITAL SAN JUAN DE DIOS. CUCUTA, COLOMBIA
}

Doctores: Alberto Duarte Contreras *, José G. Coronel ** Gilberto Bustamante ***

\section{INTRODUCCION}

Hemos hecho la presente comunicación en colaboración con los Pediatras del Servicio de Prematuros y Recién Nacidos del Hospital San Juan de Dios de Cúcuta.

Cúcuta, es capital de Departamento; tiene una población de I70.0oo habitantes; está situada a 215 metros sobre el nivel del mar, con 28 grados de temperatura media ambiental; en estas condiciones geográficas se desarrolla nuestro trabajo. Al Servicio de Maternidad del Hospital acuden las gestantes de la zona urbana, de la zona rural y las de los municipios vecinos.

\section{MATERIAL Y METODOS}

Aun cuando el Hospital San Juan de Dios de Cúcuta tiene consulta prenatal,el $86 \%$ de las parturientas que llegan al Servicio de Maternidad no han tenido dicha atención, y el 5\% llegan después de haber sido sometidas a tratamientos y maniobras de parteras empíricas. Muchas llegan en período de expulsión.

Se revisaron 3.365 Historias en el período comprendido entre el $\mathrm{I}^{\mathrm{O}}$ de noviembre de 1960 y el 20 de enero de 1963.

El Servicio de Maternidad tiene 25 camas. Para la atención hay un equipo compuesto por cinco Médicos que prestan servicio de disponibilidad de 24 horas cada uno; hay además un Médico Residente y un Médico Interno. También colaboran todos los especialistas del Hospital cuando se les llama en interconsulta. El Servicio de Enfermería lo presta una Religiosa Enfermera graduada acompañada de Auxiliares que hacen turnos de ocho horas en grupos de a seis. Tenemos una sala de trabajo adjunta a la Sala de partos y en ella hay un equipo móvil de oxigenación y reanimación.

El Servicio de Recién Nacidos y de Prematuros está atendido por dos Médicos especializados quienes cuentan

\footnotetext{
* Médico Jefe del Departamento de Obstetricia y Ginecologia, y Médico Jefe del Servicio de Maternidad del Hospital San Juan de Dios de Cúcuta. Colombia.

** Médico Jefe del Servicio de Prematuros y Recién Nacidos, y Médico Jefe del Servicio de Medicina Interna de la Clínica Infantil del Hospital San Juan de Dios. Cúcuta.

:*:* Médico Adjunto del Servicio de Prematuros y Recién Nacidos y Médico Adjunto del Servicio de Medicina Interna de la Clínica Infantil del Hosnital San Juan de Dios. Cúcuta.
} 
con la colaboración de una Religiosa y de cuatro Auxiliares. El problema de este Servicio estriba en que no tenemos ni Residente ni interno que vigile a los niños, a quienes, salvo en casos especiales, solamente se les observa en la visita pediátrica matinal; tampoco tenemos un servicio adecuado de preparación de fórmulas lácteas, y con alguna frecuencia el personal de enfermería es deficiente.

Todos los nacidos vivos pasan al Servicio de Recién Nacidos para el examen pediátrico; los nacidos con peso inferior a 2.500 gramos o los na. cidos en partos intervenidos, o los que han necesitado maniobras de reanimación, o los nacidos con problemas de isoinmunización, o los que a juicio del pediatra necesiten cuidados u observación especial, quedan retenidos ya en el Salón de Prematuros, ya en el de Recién Nacidos. Los que se consideran normales o sanos vuelven al lado de sus madres de acuerdo con el sistema "rooming in". A las madres se les da de alta en promedio a los cuatro días. Si el niño necesita un tratamiento hospitalario prolongado, la madre deja a su hijo en el Servicio.

Sistemáticamente se hace el Test de Apgar a todo recién nacido ${ }^{(1)}$. La Historia Clínica de las madres se hace por continuidad de manera que es difícil que una misma gestante tenga más de una historia; la Historia del recién nacido se adjunta a la de la madre.

\section{RESULTADOS}

Para este trabajo hemos usado la clasificación del Comité del cuidado materno infantil del Consejo de Servicios Médicos de la Asociación Médica Americana de $1959^{(2)}$.

No haremos en el presente trabajo defirenciación entre partos y abortos;

Nos hemos ceñido a las siguientes definiciones ${ }^{(3)}$ :

"Nacido vivo" es todo recién nacido que haya presentado al salir del canal del parto cualquiera de estos signos vitales: pulsación del cordón, latidos cardíacos, movimientos de músculos voluntarios y o movimientos de músculos respiratorios; el cordón ha podido ser ligado o no, y la placenta ha podido estar desprendida o no.

"Muerte fetal" a todo embarazo que haya terminado sin dar un recién nacido vivo; en éstos hemos tenido las Molas obtenidas en parto espontáneo o quirúrgico.

"Prematuro" es todo recién nacido cuyo peso al nacer sea de 2.500 gramos o menos (4) (5) (6) (i) (8).

Las ratas de mortalidad las hemos calculado con las siguientes fórmulas:

Rata de mortalidad fetal:

$\frac{\text { Muerte fetal }}{\text { Nacidos vivos + Muerte fetal }} 1.000$

Rata de muerte temprana:

$\frac{\text { Muertes tempranas }}{\text { Nacidos vivos }} 1.000$

Rata de mortalidad perinatal:

Muertes fetales + Muertes tempranas

Muertes feta?es + Nacidos vivos 
Hemos obtenido los siguientes resultados:

CUADRO 1

MORTALIDAD FETAL Y PERINATAL

\begin{tabular}{lrr}
\hline Nacidos vivos & 3.228 & \\
$\quad$ Prematuros & 316 & \\
$\quad$ Frematuros \% & & 9.78 \\
Mortalidad fetal & 180 & \\
$\quad$ Rata de mortalidad & & 52.2 \\
Antes de 28 semanas & 81 & \\
28 semanas o más & 90 & \\
$\quad$ de tiempo desconocido & 9 & \\
Muerte temprana & 47 & \\
$\quad$ Rata mortalidad & & 14.4 \\
Muerte perinatal & 227 & \\
$\quad$ Rata mortalidad & & 66.6 \\
\hline
\end{tabular}

CUADRO 2

MUERTES PERLNATALES EN RELACION CON GESTANTES QUE ASISTIERON O No a Consulta prenatal

\begin{tabular}{ccrcr}
\hline Consulta & Partos & & \multicolumn{2}{c}{$\begin{array}{c}\text { Muertes } \\
\text { perinatales }\end{array}$} \\
\hline prenetal & Total & $\%$ & Total & $\%$ \\
Asistieron & 477 & 14 & 14 & 2.9 \\
No asistieron & 2.931 & 86 & 312 & 7.2 \\
Total & 3.408 & 100 & 227 & \\
\hline
\end{tabular}

que no tuvieron este servicio rindieron un mayor tributo a la mortalidad perinatal.

CUADRO 3

MORTALIDAD PERINATAL EN RELACION CON LA EDAD DE LAS GESTANTES

\begin{tabular}{|c|c|c|c|}
\hline Edad gestantes & $\begin{array}{l}\text { Total } \\
\text { partos }\end{array}$ & $\begin{array}{c}\text { Muertes } \\
\text { perinatales }\end{array}$ & $\%$ \\
\hline Menos de 15 años & 2 & & \\
\hline De 15 a 19 años & 604 & 27 & 4.4 \\
\hline De 20 a 29 años & 1.710 & 86 & 5.0 \\
\hline De 30 a 34 años & 597 & 53 & 8.8 \\
\hline De 35 a 45 años & 483 & 52 & 10.7 \\
\hline Más de 45 años & 3 & 2 & 66.6 \\
\hline Edad desconocida & 9 & 7 & 77.7 \\
\hline Total & 3.408 & 227 & \\
\hline
\end{tabular}

Este cuadro nos muestra cómo la mortalidad perinatal aumenta progresivamente con la edad de la gestante y es mayor, en forma desproporcionada, en las mayores de cuarenta y cinco años. No tuvimos muerte perinatal en las menores de quince años.

CUADRO 4

MUERTES PERINATALES ENRELACION CON LA GRAVIDEZ

\begin{tabular}{|c|c|c|c|c|c|}
\hline Gravidez & Total partos & Fetal & $\begin{array}{r}\text { Muertes } \\
\text { Temprana }\end{array}$ & $\begin{array}{c}\text { perinatales } \\
\text { Total }\end{array}$ & $\%$ \\
\hline Grávida I & 813 & 12 & 20 & 32 & 3.9 \\
\hline Grávida II a V & 1.484 & 52 & 15 & 67 & 4.5 \\
\hline Grávida VI a X & 769 & 65 & 10 & 75 & 9.7 \\
\hline Grávida más de $\mathrm{X}$ & 297 & 49 & 1 & 59 & 16.8 \\
\hline Gravidéz desconocida & 45 & 2 & 1 & 3 & 6.6 \\
\hline Total & 3.408 & 180 & 47 & 227 & \\
\hline
\end{tabular}

Este cuadro nos muestra el reduci-

Muestra cómo la mortalidad pedo número de gestantes que tuvieron rinatal es el doble después de la graconsulta prenatal y cómo aquellas videz $\mathrm{X}$, pero a expensas de la mor- 
talidad o componente fetal, pues a medida que la paridad aumenta, sube la mortalidad fetal y baja muy visiblemente la mortalidad neonatal temprana ${ }^{(9)}$.

CUADRO 5

MORTALIDAD PERINATAL

\begin{tabular}{lr}
\hline Nacidos vivos & 3.228 \\
Muertes fetales & 180 \\
Total embarazos & 3.408 \\
Muertes neonatales tempranas & 47 \\
Total muertos niños, fetos & 227 \\
Rata de mortalidad perinatal & \\
Componente fetal & 52.2 \\
Componente muerte temprana & 14.4 \\
Total & 66.6 \\
\hline
\end{tabular}

Vemos claramente cómo el componente fetal de mortalidad perinatal es cuatro veces mayor que el componente de muerte neonatal temprana.

CUADRO 6

MUERTE FETAL SEGUN EL PESO EN GRAMOS

\begin{tabular}{lrrr}
\hline Peso en gramos & Total & $\%$ \\
\hline De 400 a 1.000 & 76 & 42.21 \\
De 1.001 a 1.500 & 5 & 2.76 \\
De 1.501 a 2.00 & 3 & 1.65 \\
De 2.001 a 2.500 & 15 & 8.32 \\
De 2.501 a 4.000 & 68 & 37.86 \\
De 4.0001 a 5.200 & 2 & 1.10 \\
De peso no especificado & 11 & 6.10 \\
\cline { 3 - 3 } & & 180 & 100.00 \\
\hline
\end{tabular}

Nos llama la atención y nos alarma el gran número de productos que obtenemos entre 400 y I.000 gramos, productos éstos que dan un tributo del ciento por ciento a la mortalidad perinatal.
Las Molas, siete en total, están incluídas en los de peso no especificado.

Hemos querido conocer la relación que pueda haber entre los recién nacidos con peso superior a los 4.000 gramos y la glicemia materna; para ello hemos investigado la curva de glicemia a 42 de estas parturientas y solamente hemos encontrado una glicemia superior a la normal en 4 pacientes o sea en el $9.5 \%$ de los casos estudiados. Citamos estas cuatro pacientes a nuevos controles: asistieron sólo dos y sus controles fueron normales. En cuanto a los recién nacidos nada especial pudimos observar durante los cuatro días del período de hospitalización. Debemos aclarar que los productos obtenidos con un peso superior a los 4.000 gramos fueron 63 repartidos así: dos mortinatos y 6I nacidos vivos. No obtuvimos productos con peso superior a los 5.200 gramos.

CUADRO 7

DISTRIBUCION PROPORCIONAL DE NEONATAL TEMPRANA SEGUN LA EDAD

\begin{tabular}{|c|c|c|}
\hline Edad & Murieron & $\%$ \\
\hline En el primer día & 19 & 40.4 \\
\hline En el segundo día & 8 & 17.0 \\
\hline En el tercer día & 6 & 12.7 \\
\hline En el cuarto dia & 5 & 10.6 \\
\hline En el quinto dia & 3 & 6.4 \\
\hline En el sexto día & 2 & 4.3 \\
\hline En el séptimo día & 4 & 8.6 \\
\hline Total & 47 & 100.0 \\
\hline
\end{tabular}

Vemos cómo la mortalidad es mayor en el primer día, $40.4 \%$, al tiempo que va descendiendo progresiva. mente en los días subsiguientes. 
Este fenómeno se presenta de manera constante desde mucho tiempo atrás muy a pesar de los adelantos en fisiopatología, clínica, terapéutica y manejo del prematuro y del recién nacido; ha sido estudiado de manera muy completa por Bundesen ${ }^{(10)}$ y en Colombia lo ha analizado detalladamente Plata Rueda ${ }^{(6)}$.

Las muertes ocurridas durante las primeras veinticuatro horas de vida se debieron unas a inmadurez respiratoria y otras a defectos en los mé- diatras las Historias de Mortalidad con el fin de mejorar conductas y técnicas. El estudio y la crítica de la Historia lo hacemos ciñéndonos a la clasificación de Kendall y Rose, aumentada en las cuatro últimas observaciones de la tercera columna por Plata Rueda de acuerdo con nuestro medio $^{(11)}{ }^{(6)}$. Al calificar la Historia de acuerdo con este cuadro podemos observar claramente si la falla está en el Obstetra, en el Pediatra, en la técnica de Enfermería o en la Institución.

CUADRO 8

CLASIFICACION DE KENDALL Y ROSE:

\begin{tabular}{lrl}
\hline A-Obstétrico I Prevenible & $1-$ Cuidado prenatal inadecuado \\
B-Pediátrico II No prevenible & $2-$ Falla familiar \\
C-Combinado III No clasificable & $3-$ Falla juicio médico \\
& $4-$ Falla técnica médico \\
& $5-$ Enfermedad intercurrente \\
& $6-$ Suceso inevitable \\
& $7-$ Insuficiencia del local \\
& $8-$ Deficiencias de dotación \\
9 & - Deficiencias de dotación \\
& $9-$ Deficiencia numérica personal \\
& $10-$ Errores técnica enfermería \\
\hline
\end{tabular}

todos de reanimación del recién nacido.

Las muertes que tuvieron como causa un traumatismo obstétrico, acaecieron en los tres primeros días.

En el séptimo día tuvimos dos muertes de causa infecciosa. Después del séptimo día no tuvimos muertes tempranas.

En las reuniones que hacemos los sábados, estudiamos junto con los Pe-
En lo que respecta a traumatismos obstétricos debemos anotar que estamos usando el fórceps (Simpson y Piper) Inicamente para rotaciones o para extracción de cabezas últimas en tanto que empleamos el Vacuum Extractor o Ventosa de Malmstrom casi con exclusividad en todos los casos de demora en el descenso o en la expulsión. Hemos tenido dos nacidos vivos con gran traumatismo obstétrico y muerte perinatal temprana atribuíbles al mal empleo de la Ventosa, pero luego con la experiencia y 
la confianza que hemos adquirido en su manejo hemos logrado éxito completo en el ciento por ciento de los 97 partos en que la hemos empleado. mordiales en la patología placentaria (desprendimientos, fibrosis, degeneraciones); ello trae consigo anoxia y muerte fetal (12) (13) (14).

CUADRO 9

CAUSAS DE MORTALIDAD PERINATAI

\begin{tabular}{|c|c|c|c|c|}
\hline \multicolumn{3}{|l|}{ Muerte fetal } & \multicolumn{2}{|l|}{ Muerte temprana } \\
\hline Causa & & $\%$ & Causa & $\%$ \\
\hline Causas y condiciones maternas & 11.7 & & Prematurez & 50 \\
\hline Enfermedad crónica & 3.3 & $(28.6) \%=$ & Traumatismo obstétrico & 6.5 \\
\hline Toxemia & 6.2 & $(52.4) \% *$ & Asfixia postnatal o atelectasias & 17.1 \\
\hline Otras & 2.2 & $(19.0) \%$ & Respiratorias & 10.6 \\
\hline $\begin{array}{l}\text { Causas de feto, placenta, } \\
\text { cordón }\end{array}$ & 46.1 & & Otras & 14.8 \\
\hline . Desprendimiento prematuro & & & & \\
\hline placenta & 19.8 & $(38.5) \% *$ & & \\
\hline $\begin{array}{l}\text { Otras alteraciones, placenta } \\
\text { Deformaciones congénitas }\end{array}$ & 14.8 & $(30.1) \% * *$ & & \\
\hline del feto & 0.2 & $(3.6) \% * *$ & & \\
\hline $\begin{array}{l}\text { Eritroblastosis e incompa- } \\
\text { tibilidades sanguíneas }\end{array}$ & 0.1 & $(1.3) \% *$ & & \\
\hline Otras & 12.1 & $(26.5) \% * *$ & & \\
\hline Desconocidas & 42.2 & & & \\
\hline
\end{tabular}

* Es porcentaje de las causas y condiciones de la madre.

** Es porcentaie de las causas en el feto, placenta, cordón.

Al estudiar la causa de las muertes fetales las agrupamos en tres capítulos: A) causas y condiciones maternas, B) causas y condiciones de feto, placenta, cordón, C) causas desconocidas.

Las causas maternas nos dan el II. $7 \%$ global. En ellas las enfermedades crónicas como diabetes mellitus, sífilis, enfermedades renales y cardiovasculares, desnutrición, anemias, parasitosis, nos dan el $28.6 \%$; y las toxemias el $52.4 \%$; o sea entre una y otra tenemos el $8 \mathbf{1} \%$ de las muertes fetales atribuibles a causa materna. Estas dos causas influyen directamente como responsables pri-
Las causas de feto, placenta, cordón, producen el $46.1 \%$ global de mortalidad fetal y de éstas, los desprendimientos prematuros de placenta dan el $38.5 \%$, y otras alteraciones de cordón y placenta dan el $30.1 \%$. Estas dos son las responsables del $68.6 \%$ de las muertes fetales por causa de feto, cordón, placenta. La incompatibilidad $\mathrm{Rh}, \mathrm{Hr}$, y $\mathrm{ABO}$ dan el I. $3 \%$ de muertes fetales de este capítulo.

El crecido número de casos del capítulo de causa desconocida 42.2\% seguramente tengan como origen un fondo morboso agudo o crónico causante o asociado a un estado anóxico 
intrauterino. Creemos poder disminuír este alto porcentaje de causas desconocidas, llevándolas a las etiológicas por intermedio de la autopsia de rutina a todos los fetos muertos, autopsia que en nuestro medio poco la practicábamos ya que hasta hace unos meses carecíamos de Laboratorio de Anatomía Patológica, agregado ésto a prejuicios muy arraigados en los padres.

El diagnóstico de prematurez lo hemos hecho basados en datos clínicos: peso, signos físicos. ${ }^{(15)}$.

El traumatismo obstétrico nos da aún un porcentaje alto $(6.5 \%)$ sobre el total de muertes tempranas y exige una revisión una revisión de conductas. Las causas respiratorias también ocupan un índice alto en la mortalidad perinatal ( $10.6 \%$ ), lo mismo que el grupo de la asfixia neonatal y atelectasias $(\mathrm{I} 7 . \mathrm{I} \%$ ); lo que nos sugiere nuevamente la inquietud de revisar nuestros métodos y prácticas de reanimación, como también el uso oportuno de oxigenoterapia y de antibióticos.

El cuadro io nos facilita agrupar rápidamente y con precisión las causas de mortalidad perinatal según haya o no componente de Anoxia.

Debemos tener presente siempre la gravedad de la anoxia, pues aun cuando no todas las veces es causante de mortalidad perinatal, sí está comprometida como causa etiológica de graves desórdenes morbosos, algunos de ellos de pronóstico sombrío tales como la parálisis cerebral, la epilepsia, los trastornos del lenguaje, los retardos mentales.
CUADRO 10

MUERTE DE NACIDOS VIVOS SEGUN HAYA PRESENCIA O AUSENCIA DE ANOXIA EN MADRE, FETO, PLACENTA O CORDON

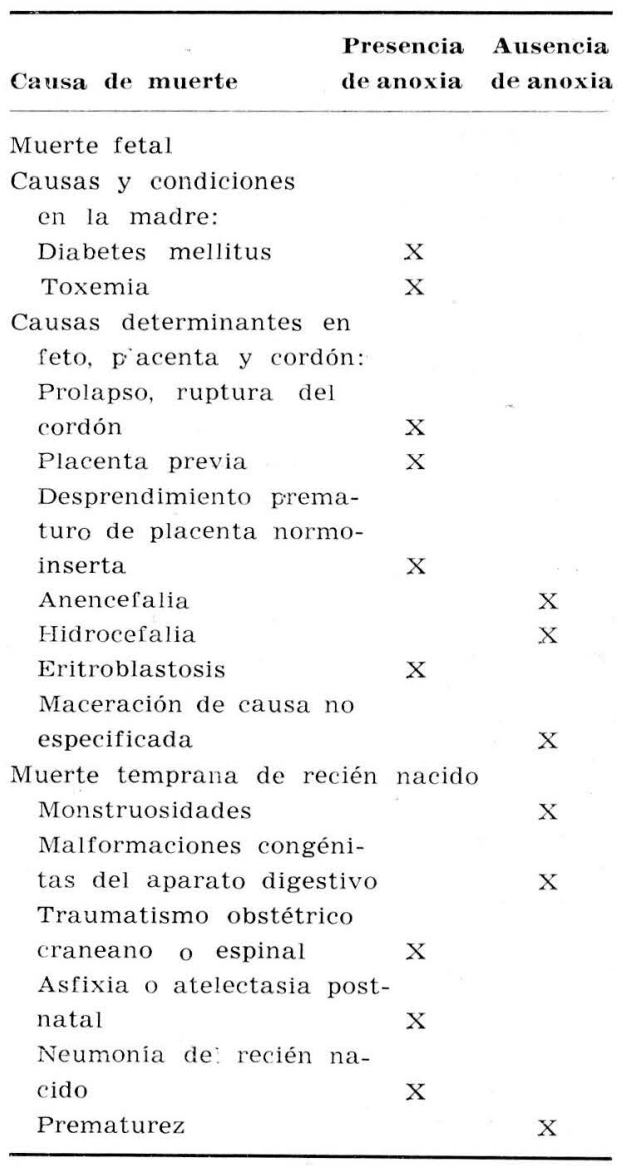

La Anoxia intrauterina puede ser la causante de la muerte fetal o de la muerte temprana, o de enfermedades durante la vida que eventualmente pueden acarrear la muerte; o de alteraciones patológicas a las que nunca se achaca una muerte eventual; o de enfermedades con recuperación completa. 
Todo esto nos muestra el peligro que lleva consigo la Anoxia y nos obliga a estar vigilando con gran solicitud a las gestantes.

Tenemos por norma en nuestro Servicio pensar siempre en el futuro del recién nacido cuando estudiamos e indicamos la conducta que se deba seguir en cada uno de los partos. temprana de niños prematuros es de $12.9 \%$. La de niños a término apenas nos alcanza al $0.2 \%$ y no tenemos mortalidad neonatal temprana en nacidos con peso superior a los 4.000 gramos. En los de peso no especificado, que corresponden todos a nacidos con peso superior a los 2.500 gramos, tampoco tuvimos mortalidad neonatal temprana.

CUADRO 11

NACIDOS VIVOS Y MUERTES FETALES ENTRE 3.408 PARTOS, DE ACUERDO CON EL PESO Y CON PRESENCIA O AUSENCIA DE COMPONNTE DE ANOXIA

\begin{tabular}{|c|c|c|c|c|c|c|c|c|c|}
\hline $\begin{array}{c}\text { Clasificación } \\
\text { por peso } \\
\text { en gramos }\end{array}$ & Total & $\begin{array}{c}\text { M u e r t e } \\
\text { Presen- } \\
\text { cia com- } \\
\text { ponente } \\
\text { Anoxia }\end{array}$ & $\begin{array}{c}\text { e fet a l } \\
\text { Ausen- } \\
\text { cia com- } \\
\text { ponente } \\
\text { Anoxia }\end{array}$ & Total & Total & $\%$ & $\begin{array}{l}\text { cidos } \\
\text { uertes te } \\
\text { Presen- } \\
\text { cia } \\
\text { Anoxia }\end{array}$ & $\begin{array}{l}\text { vivos } \\
\text { mpranas } \\
\text { Ausen- } \\
\quad \text { cia } \\
\text { Anoxia }\end{array}$ & $\begin{array}{l}\text { Sobre- } \\
\text { viven }\end{array}$ \\
\hline Total & 180 & 75 & 105 & 3228 & 47 & 1.4 & 19 & 28 & 3181 \\
\hline Prematuros & 99 & 49 & 50 & 316 & 41 & 12.9 & 14 & 27 & 275 \\
\hline 400 a 1.000 & 76 & 41 & 35 & 5 & 5 & 100 & & 5 & 0 \\
\hline 1.001 a 1.500 & 5 & 1 & 4 & 29 & 14 & 48.2 & 5 & 9 & 15 \\
\hline 1.501 a 2.000 & 3 & 1 & 2 & 88 & 19 & 21.5 & 7 & 12 & 69 \\
\hline $\begin{array}{l}2.001 \text { a } 2.500 \\
\text { A término }\end{array}$ & 15 & 61 & 9 & 149 & 3 & 1.5 & 2 & 1 & 191 \\
\hline 2.501 a 4.000 & 68 & 20 & 48 & 1824 & 6 & 0.2 & 5 & 1 & 2818 \\
\hline Sobre neso & & 2 & & & & & & & \\
\hline 4.001 a 5.200 & 2 & 2 & & 61 & & & & & 61 \\
\hline $\begin{array}{l}\text { Peso no espe- } \\
\text { cificado }\end{array}$ & 11 & & 7 & 27 & & & & & 27 \\
\hline
\end{tabular}

El siguiente cuadro, o cuadro II nos muestra cómo en un total de 3.408 partos hubo i 80 muertes fetales $y$ 3.228 nacidos vivos, de los cuales murieron tempranamente 47 .

De los 3.228 nacidos vivos, $3 \mathbf{I} 6$ nacieron con un peso inferior a 2.500 gramos, y de ellos murieron $4 \mathbf{I}$.

Vale decir; tenemos un porcentaje global de muertes neonatales tempranas de $\mathrm{I} .4 \%$. La mortalidad neonatal
El siguiente cuadro, o sea el cuadro i2 nos habla muy claramente sobre la importancia de la Anoxia, sobre todo de la Anoxia mortal, en relación con el peso del producto. Vemos cómo el componente anóxico estuvo presente el $44 \%$ de los prematuros, en tanto que en los productos a término solamente lo estuvo en el $33 \%$; muy al contrario, en los productos de sobre peso lo estuvo en el ciento por ciento. 
CUADRO 12

MUERTES PERINATALES Y RATA DE MORTALIDAD PERINATAL, EN RELACION CON EL PESO EN GRAMOS Y CON LA PRESENCIA O AUSENCIA DEL COMPONENTE DE ANOXIA. (Rata por mil)

\begin{tabular}{|c|c|c|c|c|c|c|c|c|}
\hline \multirow[b]{2}{*}{$\begin{array}{c}\text { Clasificación } \\
\text { por peso en } \\
\text { gramos }\end{array}$} & \multirow[b]{2}{*}{$\begin{array}{r}\text { Total } \\
\text { partos }\end{array}$} & \multicolumn{3}{|c|}{ Muertes perinatales } & \multicolumn{3}{|c|}{ Rata de mortalidad perinatal } & \multirow{2}{*}{$\begin{array}{c}\text { Componente } \\
\text { de Anoxia } \\
\text { de morta- } \\
\text { lidad en } \% \\
\text { mortalidad } \\
\text { perina- } \\
\text { tal total }\end{array}$} \\
\hline & & $\begin{array}{c}\text { Total } \\
\text { muertes }\end{array}$ & $\begin{array}{l}\text { Presen- } \\
\text { cia com- } \\
\text { ponente } \\
\text { Anoxia }\end{array}$ & $\begin{array}{l}\text { Ausen- } \\
\text { cia com- } \\
\text { ponente } \\
\text { Anoxia }\end{array}$ & $\begin{array}{l}\text { Presen- } \\
\text { cia com- } \\
\text { ponente } \\
\text { Anoxia }\end{array}$ & $\begin{array}{c}\text { Ausen- } \\
\text { cia com- } \\
\text { ponente } \\
\text { Anoxia }\end{array}$ & $\begin{array}{l}\text { Rata } \\
\text { sobre- } \\
\text { vivien- } \\
\text { tes }\end{array}$ & \\
\hline Total & 3408 & 227 & 94 & 133 & 27.5 & 39.0 & 933.5 & 41 \\
\hline Frematuros & 415 & 140 & 63 & 77 & 151.8 & 185.0 & 663.2 & 44 \\
\hline 400 a 1.000 & 81 & 81 & 41 & 40 & 512.2 & 187.8 & 000 & 50 \\
\hline 1.001 a 1.500 & 34 & 19 & 61 & 13 & 176.4 & 385.2 & 438.4 & 35 \\
\hline 1.501 a 2.000 & 91 & 22 & 8 & 14 & 88.8 & 155.5 & 755.7 & 38 \\
\hline 2.001 a 2.500 & 209 & 18 & 8 & 10 & 38.7 & 47.8 & 913.5 & 44 \\
\hline A término & & & & & & & & \\
\hline $\begin{array}{l}2.501 \text { a } 4.000 \\
\text { Sobre peso }\end{array}$ & 2892 & 74 & 25 & 49 & 16.4 & 28.1 & 955.5 & 33 \\
\hline 4.001 a 5.200 & 63 & 2 & 2 & & 31.7 & & 968.3 & 100 \\
\hline $\begin{array}{l}\text { Peso no espe- } \\
\text { cificado }\end{array}$ & 38 & 11 & 4 & 7 & 105.2 & 184.2 & 710.6 & 36 \\
\hline
\end{tabular}

\section{CONCLUSIONES}

Analizamos el alto índice de mortalidad fetal, con el alto tributo que a él dan el componente materno, placenta, cordón, feto y nos damos cabal cuenta de que en la mayoría de los casos se encuentra como causa un factor más o menos prevenible. Sin duda alguna si organizamos mejor nuestra consulta prenatal y aunáramos nuestro trabajo con el de las diversas consultas prenatales que funcionan en los siete centros de salud urbanos que hay en Cúcuta y con los trece de localidades vecinas, a no dudarlo disminuiría en mucho el factor materno crónico y tóxico causante de innumerables alteraciones placentarias y fetales. Para ello sería indis- pensable adiestrar el personal auxiliar de estos centros prenales y procurar que fuesen atendidos por médicos especializados. Sería indispensable además, adelantar una árdua campaña periodística, radial y personal por medio de conferencias y de educación en Colegios, en centros de trabajo, en cines, en agrupaciones, para enseñar a las futuras madres por qué y cuándo deben buscar la consulta prenatal. Y ante todo se debería hacer que la gestante al solicitar los servicios prenatales encuentre un ambiente suave, acogedor, que le preste un servicio completo y no que encuentre allí un ambiente hostil donde se le preste una mala atención, no se le instruya y se le ahuyente así de la consulta que ella busca. Este control prenatal efi- 
cientemente prestado nos disminuiría en mucho el alto índice de prematurez y haría que nuestras gestantes llegasen al parto en un mejor estado de salud y con productos de más alto peso.

La mortalidad perinatal en el Hospital San Juan de Dios de Cúcuta es aún elevada pues nos da una Rata por mil de 66.6 y esta mortalidad se hace en un 52.2 por mil a expensas de muertes intrauterinas y en un 14.4 por mil a expensas de muertes neonatales tempranas.

Nuestro índice de prematurez es de $9.78 \%$ y el índice de mortalidad perinatal temprana en prematuros es de $12.9 \%$.

Apenas un $14 \%$ de nuestra clientela hospitalaria asiste a control prenatal.

Al examinar los recién nacidos con peso superior a los 4.000 gramos, en- contramos en las madres curvas de glicemia ligeramente altas en el $9.5 \%$.

La muerte fetal fue mayor en toxémicas y en madres con alteraciones de cordón, placenta.

Las muertes neonatales tempranas fueron mayores en casos de anoxia e infecciones.

\section{RESUMEN}

Se revisan 3.365 Historias del Hospital San Juan de Dios de Cúcuta, Colombia, del período comprendido entre el $\mathrm{I}^{0}$ de noviembre de $1960 \mathrm{y}$ el 20 de enero de ig63. Ellas dan un total de 3.408 partos.

Se explican las condiciones de trabajo de nuestro Servicio.

Se estudia la mortalidad perinatal. Se dan conclusiones.

\section{BIBLIOGRAFIA}

1. APGAR V.: Proposal for new method of evaluation of newborn infant. Anesth \& Anal 35: 260-67, 1953.

2. A guide for the study of perinatal mortality and morbidity. A.M.A. 1959

3. HAROLD ABRAMSON and Col.: Resuscitation of the newborn infant. pág. 21 a 33. 1960.

4. ALBORNOZ MEDINA CARLOS y Col. Nuevos conceptos de prematuridad en nuestro medio. Rev. Col. de Pediatría. Vol. IV No 2, pág. 57-62, 1961.

5. BENICIO GAVIRIA GAVIRIA y Cols.:Letalidad perinatal. Rev. Col. Obst. y Gin. Vol XI No 3, Dág. 339, 1960.

6. PLATA RUEDA ERNESTO: Morbilidad perinatal. IV Jornadas pediátricas colombianas. Memorias, Cali. Pág. 1, 1958.

7. Plata RUEDA ERNESTO: Mortalidad neonatal hospitalaria en Bogotá. Rev. Col. Pediatría. Año XVI No 5. 417-93, 1957. 
8. CAMACHO GAMBA JORGE: Mortalidad perinatal en Colombia. Rev. Col. Pediatría. Pág. 40. Abril, 1958.

9. AMARIZ MORA ALBERTO. Edad de gestación del recién nacido en la Clínica Primero de Mavo de Bogotá. Rev. Col. Pediatría. Vol. IV No 3-4: 173-78, 1961.

10. BUNDESEN H. N.: Natal dav death J. A. M. 1953:466-73.

11. KENDALL N. and ROSE E. G.: Mechanism of studing neonatal mortality. Pediatries, 13: $476,1954$.

12. MENDOZA MARIO: Morbimortalidad del recién nacido de madre toxémica en la maternidad, en Barranquilla. Rev. Col. Pediatría Vol. III No 1-2:39-45, 1960.

13. GUTIERREz Alfonso y Cols: Mortalidad fetal en las toxemias. Rev. Col. Obst. y Gin. Vol. V No 5. Mayo 54, pág. 97.

14. PLATA RUEDA ERNESTO: Consideraciones sobre el recién nacido vivo de la toxémica. Rev. Col. Obst. y Gin. Vol. V No 5:103, 1954.

15. DELGADO CALDERON ERNESTO: Mortalidad perinatal en la operación cesárea. Rev. Col. Obst. y Gin. Vol. XII N: 5:173-87, 1961. 